\title{
Abnormal Alveolar Type 2 Cell Phenotype in AP-3 Mutant Mocha and Pearl Mice- An Electron Microscopy Study
}

\author{
Cheng-Lun Na, ChenXia Duan, Karen S. Apsley, and Timothy E. Weaver \\ Children's Hospital Research Foundation, Division Pulmonary Biology, Cincinnati, OH 45229
}

Adaptor protein-3 complex (AP-3), which consists of $\square 3, \square, \square 3, \square 3$ subunits, participates in cargoselective transport from trans Golgi to the lysosome (Dell'Angelica et al., 1998; Hurst et al., 1999). Genetic analyses using AP-3 mutant mice (Odorizzi et al., 1998; Swank et al., 2000) showed that mutations in AP-3 $\square$ (mocha) and the AP- $\square 3 \mathrm{~A}$ (pearl) genes resulted in pathogenesis similar to Hermansky Pudlak syndrome (HPS), a recessive inherited disease that is characterized by deficient lysosome functions, hypo pigmentation, prolonged bleeding time, and ceroid deposition. Pulmonary fibrosis has been demonstrated clinically in HPS and is a major cause of morbidity and mortality in these patients (Garay et al., 1978). The purpose of this study was to identify the progressive changes in the ultrastructure of lungs from mocha and pearl mice. The long term goal of this work is to determine if AP-3 mutations in alveolar type 2 cells contribute to pulmonary fibrosis.

Age matched mocha, pearl, and control mouse lungs from 3 weeks to 1 year old were fixed by immersion fixation and processed for electron microscopy as previously described (Na et al., 1998). Point counting analyses were performed to determine if AP-3 mutations affected the LB phenotype. For mocha and pearl mice, large LB (4-5 $\mu \mathrm{m}$ in diameter) were visible in alveolar type 2 cells as early as 3 weeks of age, but not in age matched controls (Figure 1a, 1b, and 1c). Inclusions filled with electron dense aggregates associated with mitochondria and LB were detected in some alveolar type 2 cells of these AP-3 mutant mice, but not in AP-3 heterozygous and wild type mice (Figure 1d). There was an increase in LB size by $90 \%$ and $50 \%$ for mocha and pearl mice respectively when compared to controls $(\mathrm{p}<0.001)$. Cryo immunogold labeling studies demonstrated that pulmonary surfactant protein $\mathrm{B}$ (SP-B) and C (SP-C) were correctly routed to multivesicular bodies and LB for the mocha and pearl mice, suggesting that trafficking of these LB proteins was not compromised by AP-3 mutations.

There was a 50\% increase in cellular Sat PC pool size $(\mathrm{p}<0.001)$, consistent with increased LB size in alveolar type 2 cells. The incidence of ceroid deposition in alveolar macrophages (AM; Figure 2a and 2b) was increased by $120 \%$ and $50 \%$ for mocha and pearl mice respectively compared to control mice $(\mathrm{p}<0.001)$. Electron dense lipid inclusions were prominent in the ciliated bronchiolar epithelium cells, but not in Clara cells. In addition, infiltration of collagen fibrils into interstitial septa and honeycomb lung infiltrated with rod-inclusion filled AM were detected in the 10 month old mocha and pearl mice, but not in younger animals.

The results of this study indicate that mutations in AP-3 gene affect alveolar type 2 cells and AM, suggesting that changes in both cell types may contribute to interstitial lung disease in these mouse models of HPS.

\section{References}

1. E. C. Dell'Angelica et al., Science 280 (1998) 431.

2. S. M. Garay et al., Am. J. Med. 66 (1979) 737.

3. J. Hirst et al., Mol. Biol. Cell. 10 (1999) 2787.

4. C.-L. Na et al., Micorsc. Microanl. 4 (Suppl. 2) (1998) 852.

5. G. Odorizzi, C. R. Coweles and S. D. Emr, Trends Cell Biol. 8 (1998) 282.

6. R. T. Swank et al., Pigment Cell Res 13 (Suppl. 8) (2000) 59. 


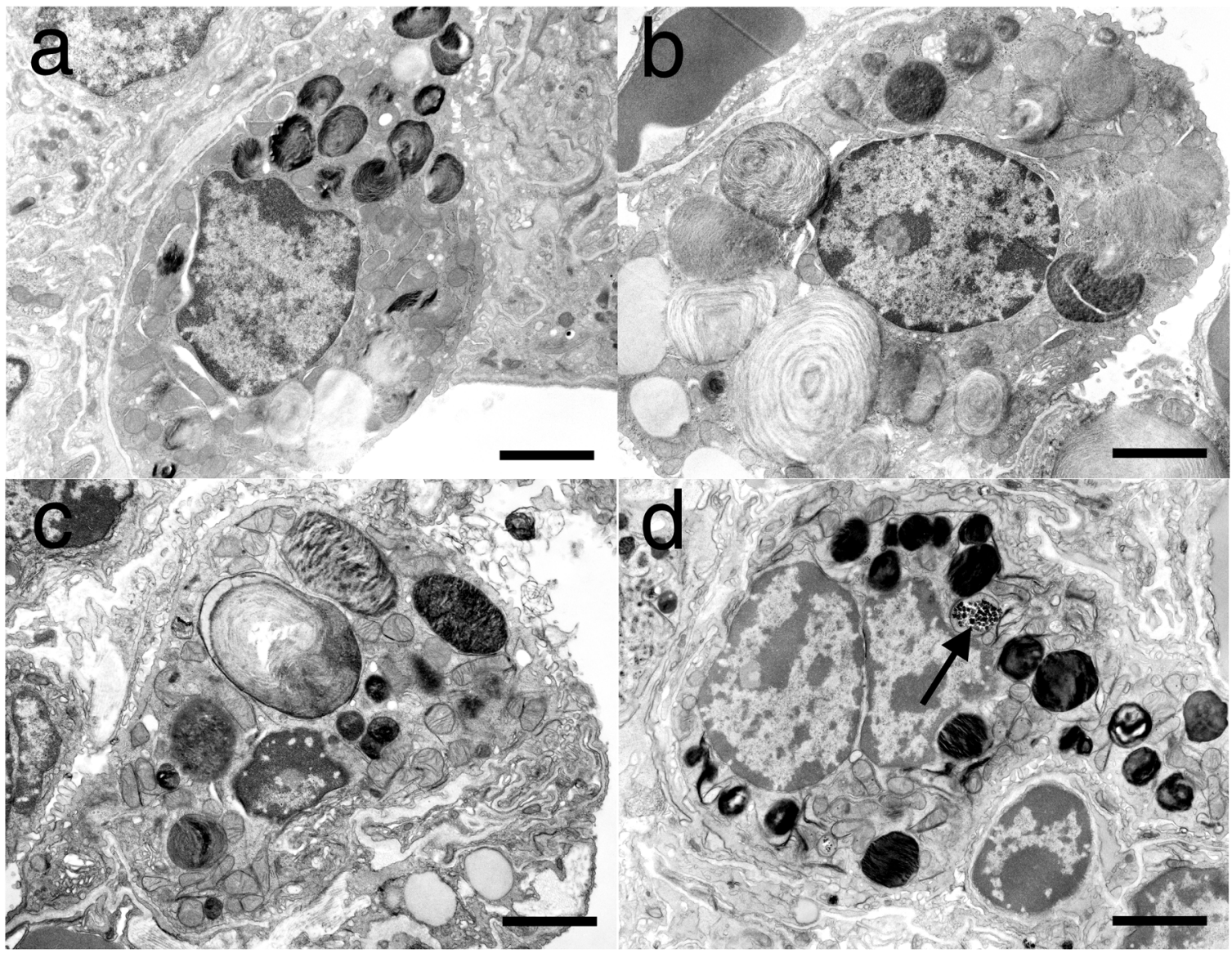

Figure 1. Alveolar type 2 cells of the control, mocha, and pearl mice. a. Control mice. b. Mocha mice. c. Pearl mice. Note that colocalization of large lamellar bodies (LB) and smaller LB in the mocha and pearl alveolar type 2 cells. d. Inclusions in alveolar type 2 cells. These inclusions (arrow), were found only in some mocha and pearl alveolar type 2 cells, but not in control alveolar type 2 cells. Scale bar is $2 \mu \mathrm{m}$.

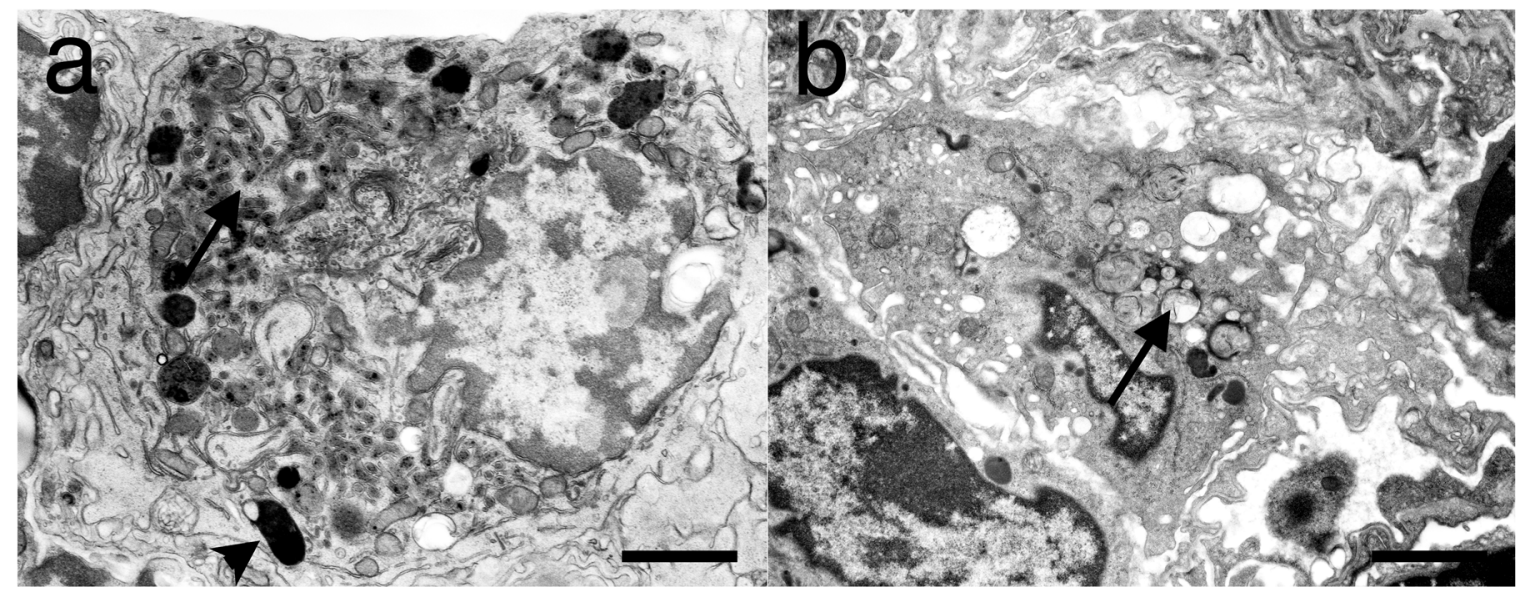

Figure 2. Alveolar macrophages. a. Mocha mice. b. Pearl mice. Electron dense melanosome-like inclusions (arrowhead) as well as ceroid pigments (arrow) were common in mocha and pearl alveolar macrophages. Scale bar is $2 \mu \mathrm{m}$. 